\title{
Truly Efficient 2-Round Perfectly Secure Message Transmission Scheme
}

\author{
Kaoru Kurosawa ${ }^{1}$ and Kazuhiro Suzuki ${ }^{2}$ \\ 1 Department of Computer and Information Sciences, Ibaraki University \\ kurosawa@mx.ibaraki.ac.jp \\ 2 Venture Business Laboratory, Ibaraki University, Hitachi, Ibaraki 316-8511, Japan \\ tutetuti@dream.com
}

\begin{abstract}
In the model of perfectly secure message transmission schemes (PSMTs), there are $n$ channels between a sender and a receiver. An infinitely powerful adversary A may corrupt (observe and forge) the messages sent through $t$ out of $n$ channels. The sender wishes to send a secret $s$ to the receiver perfectly privately and perfectly reliably without sharing any key with the receiver.

In this paper, we show the first 2-round PSMT for $n=2 t+1$ such that not only the transmission rate is $O(n)$ but also the computational costs of the sender and the receiver are both polynomial in $n$. This means that we solve the open problem raised by Agarwal, Cramer and de Haan at CRYPTO 2006.

The main novelty of our approach is to introduce a notion of pseudobasis to the coding theory. It will be an independent interest for coding theory, too.
\end{abstract}

Keywords: Perfectly secure message transmission, information theoretic security, efficiency

\section{Introduction}

In the model of ( $r$-round, $n$-channel) message transmission schemes [2], there are $n$ channels between a sender and a receiver. An infinitely powerful adversary $\mathbf{A}$ may corrupt (observe and forge) the messages sent through $t$ out of $n$ channels. The sender wishes to send a secret $s$ to the receiver in $r$-rounds without sharing any key with the receiver.

We say that a message transmission scheme is perfectly secure if it satisfies perfect privacy and perfect reliability. The perfect privacy means that the adversary A learns no information on $s$, and the perfect reliability means that the receiver can output $\hat{s}=s$ correctly.

For $r=1$, Dolev et al. showed that there exists a 1-round perfectly secure message transmission scheme (PSMT) if and only if $n \geq 3 t+1$ [2]. They also showed an efficient 1-round PSMT [2].

For $r \geq 2$, it is known that there exists a 2-round PSMT if and only if $n \geq 2 t+1[2]$. However, it is very difficult to construct an efficient scheme for 
$n=2 t+1$. Dolev et al. [2] showed a 3-round PSMT such that the transmission rate is $O\left(n^{5}\right)$, where the transmission rate is defined as

$$
\frac{\text { the total number of bits transmitted }}{\text { the size of the secrets }} \text {. }
$$

Sayeed et al. [7] showed a 2-round PSMT such that the transmission rate is $O\left(n^{3}\right)$.

Recently, Srinathan et al. showed that $n$ is a lower bound on the transmission rate of 2-round PSMT [8]. Then Agarwal, Cramer and de Haan [1] showed a 2-round PSMT such that the transmission rate is $O(n)$ at CRYPTO 2006 based on the work of Srinathan et al. $[8]^{3}$. However, the communication complexity is exponential because the sender must broadcast consistency check vectors of size 4

$$
w=\left(\begin{array}{c}
n-1 \\
t+1
\end{array}\right)=\left(\begin{array}{c}
2 t \\
t+1
\end{array}\right) .
$$

In other words, Agarwal et al. [1] achieved the transmission rate of $O(n)$ by sending exponentially many secrets. Therefore, the computational costs of the sender and the receiver are both exponential. Indeed, the authors wrote [1, Sec.6] that:

"We do not know whether a similar protocol can exist where sender and receiver restricted to polynomial time (in terms of the number of channels $n$ ) only".

In this paper, we solve this open problem. That is, we show the first 2-round PSMT for $n=2 t+1$ such that not only the transmission rate is $O(n)$ but also the computational costs of the sender and the receiver are both polynomial in $n$.

Table 1. 2-Round PSMT for $n=2 t+1$

\begin{tabular}{|c|c|c|c|c|}
\hline & Trans. rate & com. complexity & Receiver & Sender \\
\hline Agarwal et al. [1] & $O(n)$ & exponential & exponential & exponential \\
\hline This paper & $O(n)$ & $O\left(n^{3}\right)$ & poly & poly \\
\hline
\end{tabular}

The main novelty of our approach is to introduce a notion of pseudo-basis to the coding theory. Let $\mathcal{C}$ be a linear code of length $n$ over a finite field $\mathrm{F}$ with the minimum Hamming distance $d=t+1$. Consider a message transmission scheme such that the sender chooses a codeword $X_{i}=\left(x_{i 1}, \cdots, x_{i n}\right)$ of $\mathcal{C}$ randomly and

${ }^{3}$ Srinathan et al. claimed that they constructed a 2-round PSMT such that the transmission rate is $O(n)$ in [8]. However, Agarwal et al. pointed out that it has a flaw in [1].

${ }^{4}$ Indeed, in [1, page 407], it is written that "at most $O(w)$ indices and field elements are broadcast ....", where $w$ is defined in [1, page 403] as shown above. 
sends $x_{i j}$ through channel $j$ for $j=1, \cdots, n$. Note that the receiver can detect $t$ errors, but cannot correct them because $d=t+1$.

If the sender sends many codewords, however, then we can do something better. Suppose that the sender sent $X_{i}$ as shown above, and the receiver received $Y_{i}=X_{i}+E_{i}$ for $i=1, \cdots, m$, where $E_{i}$ is an error vector caused by the adversary. We now observe that the dimension of the space $\mathcal{E}$ spanned by the error vectors $E_{1}, \cdots, E_{m}$ is at most $t$ because the adversary corrupts at most $t$ channels. Suppose that $\left\{E_{i_{1}}, \cdots, E_{i_{k}}\right\}$ is such a basis, where $k \leq t$. For the same indices, we say that $\mathcal{B}=\left\{Y_{i_{1}}, \cdots, Y_{i_{k}}\right\}$ is a pseudo-basis of $\mathcal{Y}=\left\{Y_{1}, \cdots, Y_{m}\right\}$. We then show that a receiver can find a pseudo-basis $\mathcal{B}$ of $\mathcal{Y}$ in polynomial time.

By using this algorithm, we first show a 3-round PSMT for $n=2 t+1$ such that the transmission rate is $O(n)$ and the computational cost of the sender and the receiver are both polynomial in $n$. (See Fig.4.) Then combining the technique of $[8,1]$, we show a 2 -round PSMT such that not only the transmission rate is $O(n)$ but also the computational cost of the sender and the receiver are both polynomial in $n$.

(Remark) Recently, Fitzi et al. showed an efficient 2-round PSMT for $n \geq(2+\epsilon) t$ for any constant $\epsilon>0[4]$, but not for $n=2 t+1$.

\section{Main Idea}

Suppose that there are $n$ channels between the sender and the receiver, and an adversary may corrupt $t$ out of $n$ channels. We use $\mathrm{F}$ to denote $G F(p)$, where $p$ is a prime such that $p>n .{ }^{5}$

Let $\mathcal{C}$ be a linear code of length $n$ such that a codeword is $X=(f(1), \cdots, f(n))$, where $f(x)$ is a polynomial over $\mathrm{F}$ with $\operatorname{deg} f(x) \leq t$.

\subsection{Difference from Random $t$ Errors}

Consider a message transmission scheme such that the sender chooses a codeword $X=(f(1), \cdots, f(n))$ of $\mathcal{C}$ randomly, and sends $f(i)$ through channel $i$ for $i=1, \cdots, n$. Then the adversary learns no information on $f(0)$ even if she observes $t$ channels because $\operatorname{deg} f(x) \leq t$. Thus perfect privacy is satisfied.

If $n=3 t+1$, then the minimum Hamming distance of $\mathcal{C}$ is $d=n-t=2 t+1$. Hence the receiver can correct $t$ errors caused by the adversary. Thus perfect reliability is also satisfied. Therefore we can obtain a 1-round PSMT easily.

If $n=2 t+1$, however, the minimum Hamming distance of $\mathcal{C}$ is $d=n-t=$ $t+1$. Hence the receiver can only detect $t$ errors, but cannot correct them. This is the main reason why the construction of PSMT for $n=2 t+1$ is difficult.

What is a difference between usual error correction and PSMTs ? If the sender sends a single codeword $X \in \mathcal{C}$ only, then the adversary causes $t$ errors

\footnotetext{
${ }^{5}$ We adopt $G F(p)$ only to make the presentation simpler, where the elements are denoted by $0,1,2, \cdots$. But in general, our results hold for any finite field $\mathrm{F}$ whose size is larger than $n$.
} 
randomly. Hence there is no difference. If the sender sends many codewords $X_{1}, \cdots, X_{m} \in \mathcal{C}$, however, the errors are not totally random. This is because the errors always occur at the same $t$ (or less) places!

To see this more precisely, suppose that the receiver received

$$
Y_{i}=X_{i}+E_{i},
$$

where $E_{i}=\left(e_{i 1}, \cdots, e_{i n}\right)$ is an error vector caused by the adversary. Define

$$
\operatorname{support}\left(E_{i}\right)=\left\{j \mid e_{i j} \neq 0\right\} .
$$

Then there exist some $t$-subset $\left\{j_{1}, \cdots, j_{t}\right\}$ of $n$ channels such that each error vector $E_{i}$ satisfies

$$
\operatorname{support}\left(E_{i}\right) \subseteq\left\{j_{1}, \cdots, j_{t}\right\},
$$

where $\left\{j_{1}, \cdots, j_{t}\right\}$ is the set of channels that the adversary forged.

This means that the space $\mathcal{E}$ spanned by $E_{1}, \cdots, E_{m}$ has dimension at most $t$. We will exploit this fact extensively.

\subsection{Pseudo-Basis and Pseudo-Dimension}

For $i=1, \cdots, m$, suppose that the receiver received $Y_{i}$ such that

$$
Y_{i}=X_{i}+E_{i},
$$

where $X_{i} \in \mathcal{C}$ is a codeword that the sender sent and $E_{i}$ is the error vector caused by the adversary. We say that $\left\{E_{1}, \cdots, E_{m}\right\}$ is the real error-vector set of $\mathcal{Y}=\left\{Y_{1}, \cdots, Y_{m}\right\}$. We also say that $\mathcal{E}$ is the real error-vector space if it is spanned by the real error-vector set $\left\{E_{1}, \cdots, E_{m}\right\}$.

For two vectors $Y$ and $E$, we write

$$
Y=E \bmod \mathcal{C}
$$

if $Y-E \in \mathcal{C}$. In particular, eq.(3) means that

$$
Y_{i}=E_{i} \bmod \mathcal{C} .
$$

Let $\mathcal{Y}=\left\{Y_{1}, \cdots, Y_{m}\right\}$ be a set of received words. We say that $\left\{E_{1}, \cdots, E_{m}\right\}$ is an admissible error-vector set of $\mathcal{Y}$ if each $E_{i}$ satisfies $Y_{i}=X_{i}+E_{i}$ for some codeword $X_{i}$, and

$$
\left|\bigcup_{i} \operatorname{support}\left(E_{i}\right)\right| \leq t
$$

We say that $\mathcal{E}$ is an admissible error-vector space of $\mathcal{Y}$ if it is spanned by an admissible error-vector set $\left\{E_{1}, \cdots, E_{m}\right\}$.

For given $\mathcal{Y}$, an admissible error-vector set $\left\{E_{1}, \cdots, E_{m}\right\}$ may not be unique. Nevertheless, the following results holds for any admisible error-vector set.

We begin with a definition of linearly pseudo-express. 
Definition 1. We say that $Y \in \mathcal{Y}$ is linearly pseudo-expressed by $\left\{B_{1}, \cdots, B_{k}\right\}$ if there exists some $\alpha=\left(a_{1} \cdots, a_{k}\right)$ such that

$$
Y=a_{1} B_{1}+\cdots+a_{k} B_{k} \bmod \mathcal{C} .
$$

Lemma 1. Let $\left\{E_{1}, \cdots, E_{m}\right\}$ be an admissible error-vector set of $\mathcal{Y}$. Then $E_{i}$ is linearly expressed by $\left\{E_{j 1}, \cdots, E_{j k}\right\}$ if and only if $Y_{i}$ is linearly pseudo-expressed by $\left\{Y_{j 1}, \cdots, Y_{j k}\right\}$.

(Proof) Let $Y_{i}=X_{i}+E_{i}$ for each $i$, where $X_{i}$ is a codeword. Suppose that

$$
E_{i}=a_{1} E_{j 1}+\cdots+a_{k} E_{j k}
$$

for some $a_{1}, \cdots, a_{k}$. Then in $\bmod \mathcal{C}$,

$$
\begin{aligned}
& Y_{i}-\left(a_{1} Y_{j 1}+\cdots+a_{k} Y_{j k}\right) \\
= & \left(X_{i}+E_{i}\right)-a_{1}\left(X_{j 1}+E_{j 1}\right)-\cdots-a_{k}\left(X_{j 1}+E_{j 1}\right) \\
= & E_{i}-a_{1} E_{j 1}-\cdots-a_{k} E_{j k} \\
= & 0
\end{aligned}
$$

Hence $Y_{i}$ is linearly pseudo-expressed by $\left\{Y_{j 1}, \cdots, Y_{j k}\right\}$ if $E_{i}$ is linearly expressed by $\left\{E_{j 1}, \cdots, E_{j k}\right\}$. Next suppose that

$$
Y_{i}-\left(a_{1} Y_{j 1}+\cdots+a_{k} Y_{j k}\right)=0 \bmod \mathcal{C} .
$$

Then in $\bmod \mathcal{C}$,

$$
\begin{aligned}
0 & =Y_{i}-\left(a_{1} Y_{j 1}+\cdots+a_{k} Y_{j k}\right) \\
& =\left(X_{i}+E_{i}\right)-a_{1}\left(X_{j 1}+E_{j 1}\right)-\cdots-a_{k}\left(X_{j 1}+E_{j 1}\right) \\
& =E_{i}-a_{1} E_{j 1}-\cdots-a_{k} E_{j k}
\end{aligned}
$$

Hence

$$
E_{i}-a_{1} E_{j 1}-\cdots-a_{k} E_{j k} \in \mathcal{C} .
$$

From eq.(4), the Hamming weight of the left hand side is at most $t$ while the minimum Hamming weight of $\mathcal{C}$ is $t+1$. Therefore, $E_{i}-a_{1} E_{j 1}-\cdots-a_{k} E_{j k}$ is a zero-vector. Hence we obtain that

$$
E_{i}=a_{1} E_{j 1}+\cdots+a_{k} E_{j k} .
$$

This means that if $Y_{i}$ is linearly pseudo-expressed by $\left\{Y_{j 1}, \cdots, Y_{j k}\right\}$, ten $E_{i}$ is linearly expressed by $\left\{E_{j 1}, \cdots, E_{j k}\right\}$.

We next define pseudo-span.

Q.E.D.

Definition 2. We say that $\left\{Y_{j 1}, \cdots, Y_{j k}\right\} \subset \mathcal{Y}$ pseudo-spans $\mathcal{Y}$ if each $Y_{i} \in \mathcal{Y}$ can be written as

$$
Y_{i}=a_{1} Y_{j 1}+\cdots+a_{k} Y_{j k} \bmod \mathcal{C}
$$

for some $a_{i} \in \mathrm{F}$. 
We then define a pseudo-basis and the pseudo-dimension of $\mathcal{Y}$.

Definition 3. - We say that $\left\{Y_{j 1}, \cdots, Y_{j k}\right\}$ is a pseudo-basis of $\mathcal{Y}$ if it is a minimum set which pseudo-spans $\mathcal{Y}$.

- Suppose that $\left\{Y_{j 1}, \cdots, Y_{j k}\right\}$ is a pseudo-basis of $\mathcal{Y}$, where $k=\left|\left\{Y_{j 1}, \cdots, Y_{j k}\right\}\right|$. Then we say that $\mathcal{Y}$ has the pseudo-dimension $k$.

Theorem 1. Let $\left\{E_{1}, \cdots, E_{m}\right\}$ be an admissible error-vector set of $\mathcal{Y}$. Then $\mathcal{B}_{e}=\left\{E_{j 1}, \cdots, E_{j k}\right\}$ is a basis of the admisible error-vector space $\mathcal{E}$ if and only if $\mathcal{B}_{y}=\left\{Y_{j 1}, \cdots, Y_{j k}\right\}$ is a pseudo-basis of $\mathcal{Y}$. (Note that $\mathcal{B}_{e}$ and $\mathcal{B}_{y}$ have the same indices.)

In particular, the pseudo-dimension of $\mathcal{Y}$ is equal to the dimension of $\mathcal{E}$.

(Proof) Suppose that $\mathcal{B}_{e}$ is a basis of $\mathcal{E}$. That is, $\mathcal{B}_{e}$ is a minimum set which spans $\mathcal{E}$. Since $\mathcal{B}_{e}$ spans $\mathcal{E}, \mathcal{B}_{y}$ pseudo-spans $\mathcal{Y}$ from Lemma 1.

Suppose that $\mathcal{B}_{y}$ is not minimum. That is, suppose that there exists a smaller subset of $\mathcal{Y}$ which pseudo-spans $\mathcal{Y}$. Then the corresponding subset of $\left\{E_{1}, \cdots, E_{m}\right\}$ also spans $\mathcal{E}$ from Lemma 1 . However, this contradcits to the fact that $\mathcal{B}_{e}$ is minimum. Hence $\mathcal{B}_{y}$ is minimum. This shows that $\mathcal{B}_{y}$ is a pseudo-basis of $\mathcal{Y}$.

Similarly, $\mathcal{B}_{e}$ is a basis of $\mathcal{E}$ if $\mathcal{B}_{y}$ is a pseudo-basis of $\mathcal{Y}$.

Hnece the pseudo-dimension of $\mathcal{Y}$ is equal to the dimension of $\mathcal{E}$.

Since the real error-vector set is an admisible error-vector set, we obtain the following corollary from Theorem 1 .

Corollary 1. Let $\left\{E_{1}, \cdots, E_{m}\right\}$ be the real error-vector set of $\mathcal{Y}$. If $\mathcal{B}_{y}=\left\{Y_{j 1}, \cdots, Y_{j k}\right\}$ is a pseudo-basis of $\mathcal{Y}$, then $\mathcal{B}_{e}=\left\{E_{j 1}, \cdots, E_{j k}\right\}$ is a basis of the real error-vector space.

Let $\left\{E_{1}, \cdots, E_{m}\right\}$ be the real error-vector set of $\mathcal{Y}$, and let $\left\{X_{1}, \cdots, X_{m}\right\}$ be the codewords which the sender sent. Define

$$
\text { FORGED }=\bigcup_{i=1}^{m} \operatorname{support}\left(E_{i}\right) .
$$

That is, FORGED is the set of all channels that the adversary forged. Suppose that $\mathcal{B}_{y}=\left\{Y_{j 1}, \cdots, Y_{j k}\right\}$ is a pseudo-basis of $\mathcal{Y}$. Then from Corollary 1, it holds that

$$
\begin{aligned}
\text { FORGED } & =\bigcup_{i=1}^{m} \operatorname{support}\left(E_{i}\right) \\
& =\bigcup_{i=1}^{k} \operatorname{support}\left(E_{j i}\right) \\
& =\bigcup_{i=1}^{k} \operatorname{support}\left(Y_{j i}-X_{j i}\right),
\end{aligned}
$$


where eq.(5) comes from the definition of FORGED, eq.(6) holds because $\left\{E_{j 1}, \cdots, E_{j k}\right\}$ is a basis and eq. (7) holds because $Y_{j i}=X_{j i}+E_{j i}$.

The following theorem is clear since the adversary forges at most $t$ channels.

Theorem 2. The pseudo-dimension of $\mathcal{Y}$ is at most $t$.

(Proof) The dimension of the real error-vector space is at most $t$ because the adversary forges at most $t$ channels. Hence from Theorem 1, The pseudo-dimension of $\mathcal{Y}$ is at most $t$.

Q.E.D.

\subsection{How to Find Pseudo-Basis}

In this subsection, we show a polynomial time algorithm which finds the pseudodimension $k$ and a pseudo-basis $\mathcal{B}=\left\{B_{1}, \cdots, B_{k}\right\}$ of $\mathcal{Y}=\left\{Y_{1}, \cdots, Y_{m}\right\}$.

Theorem 1 shows that $\mathcal{B}_{y}=\left\{Y_{j 1}, \cdots, Y_{j k}\right\}$ is a pseudo-basis of $\mathcal{Y}$ if and only if $\mathcal{B}_{e}=\left\{E_{j 1}, \cdots, E_{j k}\right\}$ is a basis of an admisible error-vector space $\mathcal{E}$, where $\left\{E_{1}, \cdots, E_{m}\right\}$ is the admissible error-vector set. On the other hand, we can find a basis of a vector space easily by using a greedy algorithm as shown in Fig.1. This means that a pseudo-basis $\mathcal{B}$ can be found by using a similar greedy algorithm.

Fig. 1. How to Find a Basis of $\mathcal{E}$

$$
\begin{aligned}
& \text { Input: }\left\{E_{1}, \cdots, E_{m}\right\} \text {. } \\
& \text { 1. Let } i=1 \text { and } \mathcal{B}=\emptyset \text {. } \\
& \text { 2. While } i \leq m \text { and }|\mathcal{B}|<t \text {, do: } \\
& \text { (a) Check if } Y_{i} \text { is linearly expressed by } \mathcal{B} \text {. } \\
& \text { If NO, then add } E_{i} \text { to } \mathcal{B} \text {. } \\
& \text { (b) Let } i \leftarrow i+1 \text {. } \\
& \text { 3. Output } \mathcal{B} \text { as a basis and } k=|\mathcal{B}| \text { as the dimension. }
\end{aligned}
$$

Remember that $Y$ is linearly pseudo-expressed by $\left\{B_{1}, \cdots, B_{k}\right\}$ if there exists some $\alpha=\left(a_{1} \cdots, a_{k}\right)$ such that

$$
X(\alpha)=Y-\left(a_{1} B_{1}+\cdots+a_{k} B_{k}\right) \in \mathcal{C}
$$

Let

$$
X(\alpha)=\left(x_{1}(\alpha), \cdots, x_{n}(\alpha)\right) .
$$

Then it is clear that $x_{j}(\alpha)$ is a linear expression of $\left(a_{1} \cdots, a_{k}\right)$ from eq.(8).

In Fig.2, we show a polynomial time algorithm which checks if $Y$ is linearly pseudo-expressed by $\left\{B_{1}, \cdots, B_{k}\right\}$. It is easy to see that each coefficient of $f_{\alpha}(x)$ is a linear expression of $\left(a_{1} \cdots, a_{k}\right)$. Hence at step $3, f_{\alpha}(j)=x_{j}(\alpha)$ is a linear 
equation on $\left(a_{1} \cdots, a_{k}\right)$. It is now clear that the algorithm of Fig.2 outputs YES if and only if $X(\alpha) \in \mathcal{C}$ for some $\alpha$. Hence it outputs YES if and only if $Y$ is linearly pseudo-expressed by $\left\{B_{1}, \cdots, B_{k}\right\}$.

Fig. 2. How to Check if $Y$ is linearly pseudo-expressed by $\mathcal{B}$

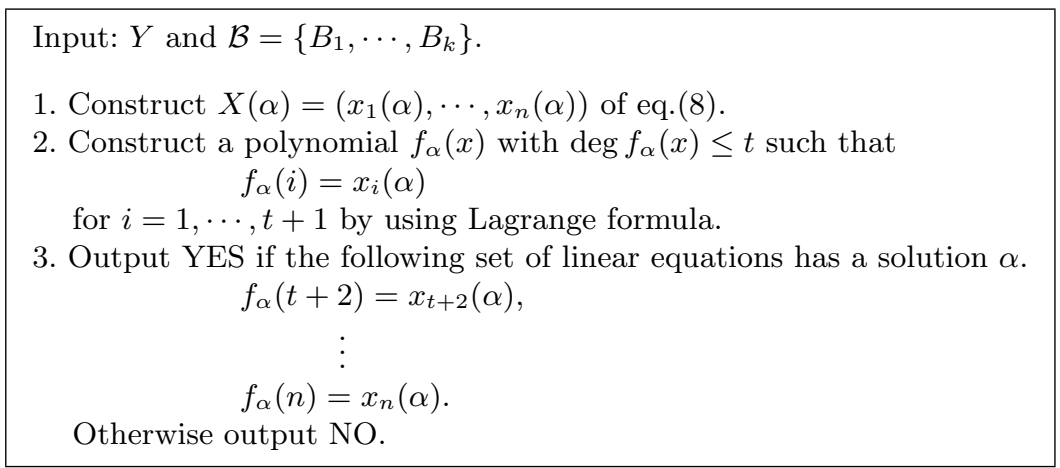

In Fig.3, we show a polynomial time algorithm which finds the pseudodimension $k$ and a pseudo-basis $\mathcal{B}=\left\{B_{1}, \cdots, B_{k}\right\}$ of $\mathcal{Y}=\left\{Y_{1}, \cdots, Y_{m}\right\}$. Note that Fig.3 is almost the same as Fig.1. Indeed, it is obtained by replacing $E_{i}$ of Fig.1 with $Y_{i}$. We call the algorithm of Fig.1 the real-basis finding algorithm, and call the algorithm of Fig.3 the pseudo-basis finding algorithm

Let $\left\{E_{1}, \cdots, E_{m}\right\}$ be an admissble error-vector set of $\mathcal{Y}$, and let $\mathcal{E}$ be the vector space spanned by $\left\{E_{1}, \cdots, E_{m}\right\}$. Supppose that we apply the real-basis finding algorithm to $\left\{E_{1}, \cdots, E_{m}\right\}$, and apply the pseudo-basis finding algorithm to $\mathcal{Y}=\left\{Y_{1}, \cdots, Y_{m}\right\}$. The real-basis finding algorithm outputs a basis $\mathcal{B}^{\prime}$ of $\mathcal{E}$. We will show that the pseudo-basis finding algorithm outputs a pseudo-basis $\mathcal{B}$ of $\mathcal{Y}$.

Step 2(a) is the only difference between the two algorithms. Further from Lemma $1, Y_{i}$ is added to $\mathcal{B}$ at step $2(\mathrm{a})$ if and only if $E_{i}$ is added to $\mathcal{B}^{\prime}$ at step 2(a). Hence the pseudo-basis finding algorithm behaves in the same way as the real-basis finding algorithm. In particular, if the real-basis finding algorithm outputs $\mathcal{B}^{\prime}=\left\{E_{j 1}, \cdots, E_{j k}\right\}$, then the pseudo-basis finding algorithm outputs $\mathcal{B}=\left\{Y_{j 1}, \cdots, Y_{j k}\right\}$. Therefore $\mathcal{B}$ is a pseudo-basis of $\mathcal{Y}$ from Theorem 1 because $\mathcal{B}^{\prime}$ is a basis of $\mathcal{E}$.

\subsection{Broadcast}

We say that a sender (receiver) broadcasts $x$ if it she sends $x$ over all $n$ channels. Since the adversary corrupts at most $t$ out of $n=2 t+1$ channels, the receiver (sender) receives $x$ correctly from at least $t+1$ channels. Therefore, the receiver (sender) can accept $x$ correctly by taking the majority vote. 
Fig. 3. How to Find a Pseudo-Basis $\mathcal{B}$ of $\mathcal{Y}$

Input: $\mathcal{Y}=\left\{Y_{1}, \cdots, Y_{m}\right\}$.

1. Let $i=1$ and $\mathcal{B}=\emptyset$.

2. While $i \leq m$ and $|\mathcal{B}|<t$, do:

(a) Check if $Y_{i}$ is linearly pseudo-expressed by $\mathcal{B}$ by using Fig.2. If $\mathrm{NO}$, then add $Y_{i}$ to $\mathcal{B}$.

(b) Let $i \leftarrow i+1$.

3. Output $\mathcal{B}$ as a pseudo-basis and $k=|\mathcal{B}|$ as the pseudo-dimension.

\subsection{How to Apply to 3-Round PSMT}

We now present an efficient 3-round PSMT for $n=2 t+1$ in Fig.4.

Fig. 4. Our 3-round PSMT for $n=2 t+1$

The sender wishes to send $\ell=n t$ secrets $s_{1}, \cdots, s_{\ell} \in \mathrm{F}$ to the receiver.

1. The sender sends a random codeword $X_{i}=\left(f_{i}(1), \cdots, f_{i}(n)\right)$, and the receiver receives $Y_{i}=X_{i}+E_{i}$ for $i=1, \cdots, \ell+t$, where $\operatorname{deg} f_{i}(x) \leq t$ and $E_{i}$ is the error vector caused by the adversary.

2. The receiver finds a pseudo-basis $\mathcal{B}=\left\{Y_{j 1}, \cdots, Y_{j k}\right\}$, where $k \leq t$, by using the algorithm of Fig.3.

He then broadcasts $\mathcal{B}$ and $\Lambda_{\mathcal{B}}=\left\{j_{1}, \cdots, j_{k}\right\}$.

3. The sender constructs FORGED of eq.(7) from $\left\{E_{j}=Y_{j}-X_{j} \mid j \in \Lambda_{\mathcal{B}}\right\}$, encrypts $s_{1}, \cdots, s_{\ell}$ by using $\left\{f_{i}(0) \mid i \notin \Lambda_{\mathcal{B}}\right\}$ as the key of one-time pad, and then broadcasts FORGED and the ciphertexts.

4. The receiver reconstructs $f_{i}(x)$ by ignoring all channels of FORGED, and applying Lagrange formula to the remaining elements of $Y_{i}$. He then decrypts the ciphertexts by using $\left\{f_{i}(0) \mid i \notin \Lambda_{\mathcal{B}}\right\}$.

Further by combining the technique of $[8,1]$, we can construct a 2-round PSMT such that not only the transmission rate is $O(n)$, but also the computational cost of the sender and the receiver are both polynomial in $n$. The details will be given in the following sections.

\section{Details of Our 3-Round PSMT}

In this section, we describe the details of our 3-round PSMT for $n=2 t+1$ which was outlined in Sec.2.5, and prove its security. We also show that the 
transmission rate is $O(n)$ and the computational cost of the sender and the receiver are both polynomial in $n$.

Remember that FORGED is the set of all channels which the adversary forged, and "broadcast" is defined in Sec.2.4.

\subsection{3-round Protocol for $n=2 t+1$}

The sender wishes to send $\ell=n t$ secrets $s_{1}, \cdots, s_{\ell} \in \mathrm{F}$ to the receiver.

Step 1. The sender does the following for $i=1,2, \cdots, t+\ell$.

1. She chooses a polynomial $f_{i}(x)$ over $\mathrm{F}$ such that $\operatorname{deg} f_{i}(x) \leq t$ randomly. Let $X_{i}=\left(f_{i}(1), \cdots, f_{i}(n)\right)$.

2. She send $f_{i}(j)$ through channel $j$ for $j=1, \cdots, n$.

The receiver then receives $Y_{i}=X_{i}+E_{i}$, where $E_{i}$ is the error vector caused by the adversary.

Step 2. The receiver does the following.

1. Find the pseudo-dimension $k$ and a pseudo-basis $\mathcal{B}=\left\{Y_{j 1}, \cdots, Y_{j k}\right\}$ of $\left\{Y_{1}, \cdots, Y_{t+\ell}\right\}$ by using the algorithm of Fig.3.

2. Broadcast $k, \mathcal{B}$ and $\Lambda_{\mathcal{B}}=\left\{j_{1}, \cdots, j_{k}\right\}$. where $\Lambda_{\mathcal{B}}$ is the set of indices of $\mathcal{B}$.

Step 3. The sender does the following.

1. Construct FORGED of eq.(7) from $\left\{E_{j}=Y_{j}-X_{j} \mid j \in \Lambda_{\mathcal{B}}\right\}$.

2. Compute $c_{1}=s_{1}+f_{i_{1}}(0), \cdots, c_{\ell}=s_{\ell}+f_{i_{\ell}}(0)$ for $i_{1}, \cdots, i_{\ell} \notin \Lambda_{\mathcal{B}}$.

3. Broadcast FORGED and $\left(c_{1}, \cdots, c_{\ell}\right)$.

Step 4. The receiver does the following. Let $Y_{i}=\left(y_{i 1}, \cdots, y_{i n}\right)$.

1. For each $i \notin \Lambda_{\mathcal{B}}$, find a polynomial $f_{i}^{\prime}(x)$ with $\operatorname{deg} f_{i}^{\prime}(x) \leq t$ such that

$$
f_{i}^{\prime}(j)=y_{i, j}
$$

for all $j \notin$ FORGED.

2. Compute $s_{1}^{\prime}=c_{1}-f_{i_{1}}^{\prime}(0), \cdots, s_{\ell}^{\prime}=c_{\ell}-f_{i_{\ell}}^{\prime}(0)$ for $i_{1}, \cdots, i_{\ell} \notin \Lambda_{\mathcal{B}}$.

3. Output $\left(s_{1}^{\prime}, \cdots, s_{\ell}^{\prime}\right)$.

\subsection{Security}

We first prove the perfect privacy. Consider $f_{i}(x)$ such that $i \notin \Lambda_{\mathcal{B}}$. For such $i$, $Y_{i}$ is not broadcast at step 2-2. Hence the adversary observes at most $t$ elements of $\left(f_{i}(1), \cdots, f_{i}(n)\right)$. This means that she has no information on $f_{i}(0)$ because $\operatorname{deg} f_{i}(x) \leq t$. Therefore since $\left\{f_{i}(0) \mid i \notin \Lambda_{\mathcal{B}}\right\}$ is used as the key of one-time-pad, the adversary learns no information on $s_{1}, \cdots, s_{\ell}$.

We next prove the perfect reliability. We first show that there exist $\ell$ indices $i_{1}, i_{2}, \cdots, i_{\ell}$ such that

$$
\left\{i_{1}, i_{2}, \cdots, i_{\ell}\right\} \subseteq\{1,2, \cdots, t+\ell\} \backslash \Lambda_{\mathcal{B}}
$$


This is because

$$
t+\ell-\left|\Lambda_{\mathcal{B}}\right| \geq t+\ell-t=\ell
$$

from Theorem 2. We next show that $f_{i}^{\prime}(x)=f_{i}(x)$ for each $i \notin \Lambda_{\mathcal{B}}$ at Step 4 . This is because

$$
f_{i}^{\prime}(j)=y_{i, j}=x_{i, j}=f_{i}(j)
$$

for all $j \notin$ FORGED, and

$$
n-\mid \text { FORGED } \mid \geq 2 t+1-t \geq t+1 \text {. }
$$

Also note that $\operatorname{deg} f_{i}(x) \leq t$ and $\operatorname{deg} f_{i}^{\prime}(x) \leq t$. Therefore $s_{i}^{\prime}=s_{i}$ for $i=1, \cdots, \ell$.

\subsection{Efficiency}

Let $|\mathrm{F}|$ denote the bit length of the field elements. Let COM(i) denote the communication complexity of Step $i$ for $i=1,2,3$. Then

$$
\begin{aligned}
& \operatorname{COM}(1)=O(n(t+\ell))|\mathrm{F}|)=O(n \ell|\mathrm{F}|), \\
& \operatorname{CoM}(2)=O\left(n^{2} t|\mathrm{~F}|\right)=O(n \ell|\mathrm{F}|), \\
& \operatorname{com}(3)=O\left(n \ell|\mathrm{F}|+t n \log _{2} n\right)=O(n \ell|\mathrm{F}|)
\end{aligned}
$$

since $\ell=n t$. Hence the total communication complexity is $O(n \ell|\mathrm{F}|)=O\left(n^{3}|\mathrm{~F}|\right)$. Further the sender sends $\ell$ secrets $s_{1}, \cdots, s_{\ell} \in \mathrm{F}$. Therefore, the transmission rate is $O(n)$ because

$$
\frac{n \ell|\mathrm{F}|}{\ell|\mathrm{F}|}=n .
$$

It is easy to see that the computational costs of the sender and the receiver are both polynomial in $n$.

\section{Our Basic 2-Round PSMT}

In this section, we show our basic 2-round PSMT for $n=2 t+1$ such that the transmission rate is $O\left(n^{2} t\right)$ and the computational costs of the sender and the receiver are both polynomial in $n$.

For two vectors $U=\left(u_{1}, \cdots, u_{n}\right)$ and $Y=\left(y_{1}, \cdots, y_{n}\right)$, define

$$
\begin{aligned}
& d_{u}(U, Y)=\left\{u_{j} \mid u_{j} \neq y_{j}\right\} \\
& d_{I}(U, Y)=\left\{j \mid u_{j} \neq y_{j}\right\} .
\end{aligned}
$$

Remember that $\mathcal{C}$ is the set of all $(f(1), \cdots, f(n))$ such that $\operatorname{deg} f(x) \leq t$.

\subsection{Randomness Extractor}

Suppose that the adversary has no information on $\ell$ out of $m$ random elements $r_{1}, \cdots, r_{m} \in \mathrm{F}$. In this case, let $R(x)$ be a polynomial with $\operatorname{deg} R(x) \leq m-1$ such that $R(i)=r_{i}$ for $i=1, \cdots, m$. Then it is well known [1, Sec.2.4] that the adversary has no information on

$$
z_{1}=R(m+1), \cdots, z_{\ell}=R(m+\ell) .
$$




\subsection{Basic 2-round Protocol}

The sender wishes to send a secret $s \in \mathrm{F}$ to the receiver.

Step 1. The receiver does the following for $i=1,2, \ldots, n$.

1. He chooses a random polynomial $f_{i}(x)$ such that $\operatorname{deg} f_{i}(x) \leq t$.

2. He sends

$$
X_{i}=\left(f_{i}(1), \cdots, f_{i}(n)\right)
$$

through channel $i$, and the sender receives

$$
U_{i}=\left(u_{i 1}, \ldots, u_{i n}\right)
$$

3. Through each channel $j$, he sends $f_{i}(j)$ and the sender receives

$$
y_{i j}=f_{i}(j)+e_{i j}
$$

where $e_{i j}$ is the error caused by the adversary. Let

$$
Y_{i}=\left(y_{i 1}, \cdots, y_{i n}\right), E_{i}=\left(e_{i 1}, \cdots, e_{i n}\right)
$$

Step 2. The sender does the following.

1. For $i=1, \cdots, n$,

(a) If $u_{i i} \neq y_{i i}$ or $\left|d_{u}\left(U_{i}, Y_{i}\right)\right| \geq t+1$ or $U_{i} \notin \mathcal{C}$,

then broadcast "ignore channel $i$ ". ${ }^{6}$

This channel will be ignored from now on because it is forged clearly.

(b) Else define $r_{i}$ as

$$
r_{i}=u_{i i}=y_{i i}
$$

2. Find a polynomial $R(x)$ with $\operatorname{deg} R(x) \leq n-1$ such that

$$
R(i)=r_{i}
$$

for each $i$.

3. Compute $R(n+1)$ and broadcast

$$
c=s+R(n+1) \text {. }
$$

4. Find the pseudo-dimension $k$ and a pseudo-basis $\mathcal{B}=\left\{Y_{j 1}, \cdots, Y_{j k}\right\}$ of $\left\{Y_{1}, \cdots, Y_{n}\right\}$ by using the algorithm of Fig.3.

Broadcast $k, \mathcal{B}$ and $\Lambda_{\mathcal{B}}=\left\{j_{1}, \cdots, j_{k}\right\}$.

5. Broadcast $d_{u}\left(U_{i}, Y_{i}\right)$ and $d_{I}\left(U_{i}, Y_{i}\right)$ for each $i$.

Step 3. The receiver does the following.

1. Construct FORGED of eq.(7) from $\left\{E_{i}=Y_{i}-X_{i} \mid i \in \Lambda_{\mathcal{B}}\right\}$.

\footnotetext{
${ }^{6}$ For simplicity, we assume that there are no such channels in what follows.
} 
2. For each $i$, find a polynomial $u_{i}(x)$ with $\operatorname{deg} u_{i}(x) \leq t$ such that

$$
\begin{aligned}
& u_{i}(j)=u_{i j} \text { for all } j \in d_{I}\left(U_{i}, Y_{i}\right), \\
& u_{i}(j)=f_{i}(j) \text { for all } j \text { such that } j \notin d_{I}\left(U_{i}, Y_{i}\right) \text { and } j \notin \text { FORGED }
\end{aligned}
$$

3. Find a polynomial $R^{\prime}(x)$ with $\operatorname{deg} R^{\prime}(x) \leq n-1$ such that

$$
R^{\prime}(i)=u_{i}(i)
$$

for each $i .^{7}$

4. Compute $R^{\prime}(n+1)$ and output

$$
s^{\prime}=c-R^{\prime}(n+1) .
$$

\subsection{Security}

We first prove the perfect privacy.

Lemma 2. There is at least one $r_{i}$ on which the adversary has no information.

Proof. Consider a non-corrupted channel $i$ such that $i \notin \Lambda_{\mathcal{B}}$. First the sender does not broadcast $r_{i}$ at step $2-4$ because $i \notin \Lambda_{\mathcal{B}}$. Next because $f_{i}(i)$ is sent through channel $i$ that the adversary does not corrupt, we have

$$
r_{i}=u_{i i}=f_{i}(i) .
$$

Further the adversary observes at most $t$ values of $\left(f_{i}(1), \cdots, f_{i}(n)\right)$. Hence the adversary has no information on $r_{i}=f_{i}(i)$ because $\operatorname{deg} f_{i}(x) \leq t$.

Finally there exists at least one non-corrupted channel $i$ such that $i \notin \Lambda_{\mathcal{B}}$ because

$$
n-t-\left|\Lambda_{\mathcal{B}}\right| \geq n-2 t=1 \text {. }
$$

Therefore, the adversary has no information on $R(n+1)$ from Sec.4.1. Hence she learns no information on $s$ from $c=s+R(n+1)$.

We next prove the perfect reliability. If $j \notin$ FORGED and $j \notin d_{I}\left(U_{i}, Y_{i}\right)$, then $f_{i}(j)=y_{i j}=u_{i j}$ from the definition of $d_{I}\left(U_{i}, Y_{i}\right)$. Therefore, at step 3-2,

$$
u_{i}(j)=u_{i j}
$$

for all $j \in d_{I}\left(U_{i}, Y_{i}\right)$, and for all $j$ such that $j \notin d_{I}\left(U_{i}, Y_{i}\right)$ and $j \notin$ FORGED. This means that $u_{i}(j)=u_{i j}$ for each $j \in\left(\overline{\text { FORGED }} \cup d_{I}\left(U_{i}, Y_{i}\right)\right)$, where

$$
\left.\mid \overline{\text { FORGED }} \cup d_{I}\left(U_{i}, Y_{i}\right)\right)|\geq| \overline{\text { FORGED }} \mid \geq n-t=(2 t+1)-t=t+1 .
$$

\footnotetext{
7 "for each $i$ " can be replaced by "for each $i \notin \Lambda_{\mathcal{B}}$ " at step 2-2 and step 3-3.
} 
Further since $\operatorname{deg} u_{i}(x) \leq t$ and $U_{i} \in \mathcal{C}$, it holds that

$$
\left(u_{i}(1), \cdots, u_{i}(n)\right)=\left(u_{i 1}, \cdots, u_{i n}\right) .
$$

In particular, $u_{i}(i)=u_{i i}$. Therefore from eq.(9), we have that

$$
R(i)=r_{i}=u_{i i}=u_{i}(i)=R^{\prime}(i)
$$

for each $i$. Hence we obtain that $R^{\prime}(x)=R(x)$ because $\operatorname{deg} R^{\prime}(x) \leq n-1$ and $\operatorname{deg} R(x) \leq n-1$. Consequently,

$$
s^{\prime}=c-R^{\prime}(n+1)=c-R(n+1)=s .
$$

Thus the receiver can compute $s^{\prime}=s$ correctly.

\subsection{Efficiency}

Let COM(i) denote the communication complexity of Step $i$ for $i=1,2$. Note that $\left|d_{u}\left(U_{i}, Y_{i}\right)\right|=\left|d_{I}\left(U_{i}, Y_{i}\right)\right| \leq t$ for each $i$. Then

$$
\begin{aligned}
\operatorname{COM}(1)= & O(n(n+n)|\mathrm{F}|)=O\left(n^{2}|\mathrm{~F}|\right) \\
\operatorname{COM}(2)= & O\left(\left(\left|d_{I}\left(U_{i}, Y_{i}\right)\right| \log _{2} n+\left|d_{u}\left(U_{i}, Y_{i}\right)\right||\mathrm{F}|\right) n^{2}\right. \\
& \left.+\left(\log _{2} n+n|\mathcal{B}||\mathrm{F}|+\left|\Lambda_{\mathcal{B}}\right| \log _{2} n\right) n+|\mathrm{F}| n\right) \\
= & O\left(t n^{2} \log _{2} n+t n^{2}|\mathrm{~F}|+n \log _{2} n+n^{2} t|\mathrm{~F}|+t n \log _{2} n+|\mathrm{F}| n\right) \\
= & O\left(n^{2} t|\mathrm{~F}|\right)
\end{aligned}
$$

because $|\mathcal{B}|=\left|\Lambda_{\mathcal{B}}\right| \leq t$. Hence the total communication complexity is $O\left(n^{2} t|\mathrm{~F}|\right)$. The transmission rate is $O\left(n^{2} t\right)$ because the sender sends one secret.

It is easy to see that the computational cost of the sender and the receiver are polynomial in $n$.

\section{More Efficient 2-Round Protocol}

In our basic 2-round protocol, the sender sends a single secret. In this section, we show a more efficient 2-round protocol such that the sender sends $t^{2}$ secrets by running the basic protocol $t$ times in parallel. This implies that we can reduce the transmission rate from $O\left(n^{2} t\right)$ to $O\left(n^{2}\right)$.

\subsection{Protocol}

The sender wishes to send $\ell=t^{2}$ secrets $s_{1}, s_{2}, \ldots, s_{\ell} \in \mathrm{F}$ to the receiver.

Step 1. The receiver does the following for each channel $i$.

For $h=0,1, \cdots, t-1$;

1. He chooses a random polynomial $f_{i+h n}(x)$ such that $\operatorname{deg} f_{i+h n}(x) \leq t$. 
2. He sends

$$
X_{i+h n}=\left(f_{i+h n}(1), \cdots, f_{i+h n}(n)\right)
$$

through channel $i$, and the sender receives

$$
U_{i+h n}=\left(u_{i+h n, 1}, \cdots, u_{i+h n, n}\right)
$$

3. Through each channel $j$, he sends $f_{i+h n}(j)$ and the sender receives

$$
y_{i+h n, j}=f_{i+h n}(j)+e_{i+h n, j},
$$

where $e_{i+h n, j}$ is the error caused by the adversary. Let

$$
Y_{i+h n}=\left(y_{i+h n, 1}, \cdots, y_{i+h n, n}\right), E_{i+h n}=\left(e_{i+h n, 1}, \cdots, e_{i+h n, n}\right) .
$$

Step 2. The sender does the following.

1. Find the pseudo-dimension $k$ and a pseudo-basis $\mathcal{B}=\left\{Y_{j 1}, \ldots, Y_{j k}\right\}$ of $\left\{Y_{1}, \cdots, Y_{t n}\right\}$ by using the algorithm of Fig.3.

Broadcast $k, \mathcal{B}$ and $\Lambda_{\mathcal{B}}=\left\{j_{1}, \cdots, j_{k}\right\}$.

2. For $i=1, \cdots, n$,

(a) If $u_{i+h n, i} \neq y_{i+h n, i}$ or $\left|d_{u}\left(U_{i+h n}, Y_{i+h n}\right)\right| \geq t+1$

or $U_{i+h n} \notin \mathcal{C}$ for some $h$, then broadcast "ignore channel $i$ ". 8

This channel will be ignored from now on because it is forged clearly.

(b) Else define $r_{i+h n}$ as

$$
r_{i+h n}=u_{i+h n, i}=y_{i+h n, i}
$$

for $h=0, \cdots, t-1$.

3. Find a polynomial $R(x)$ with $\operatorname{deg} R(x) \leq n t-1$ such that

$$
R(i+h n)=r_{i+h n}
$$

for each $i+h n$.

4. Compute $R(n t+1), \cdots, R(n t+\ell)$ and broadcast

$$
c_{1}=s_{1}+R(n t+1), \cdots, c_{\ell}=s_{\ell}+R(n t+\ell) .
$$

5. Broadcast $d_{u}\left(U_{i+h n}, Y_{i+h n}\right)$ and $d_{I}\left(U_{i+h n}, Y_{i+h n}\right)$ for each $i+h n$.

Step 3. The receiver does the following.

1. Construct FORGED of eq.(7) from $\left\{E_{i}=Y_{i}-X_{i} \mid i \in \Lambda_{\mathcal{B}}\right\}$.

2. For each $i+h n$, find a polynomial $u_{i+h n}(x)$ with $\operatorname{deg} u_{i+h n}(x) \leq t$ such that $u_{i+h n}(j)=u_{i+h n, j}$ for all $j \in d_{I}\left(U_{i+h n}, Y_{i+h n}\right)$

$u_{i+h n}(j)=f_{i+h n}(j)$ for all $j$ such that $j \notin d_{I}\left(U_{i+h n}, Y_{i+h n}\right)$ and $j \notin$ FORGED

3. Find a polynomial $R^{\prime}(x)$ with $\operatorname{deg} R^{\prime}(x) \leq n t-1$ such that

$$
R^{\prime}(i+h n)=u_{i+h n}(i)
$$

for each $i+h n$. $^{9}$

4. Compute $R^{\prime}(n t+1), \cdots, R^{\prime}(n t+\ell)$ and output

$$
s_{1}^{\prime}=c_{1}-R^{\prime}(n t+1), \cdots, s_{\ell}^{\prime}=c_{\ell}-R^{\prime}(n t+\ell) .
$$

\footnotetext{
${ }^{8}$ For simplicity, we assume that there are no such channels in what follows.

9 "for each $i+h n$ " can be replaced by "for each $i+h n \notin \Lambda_{\mathcal{B}}$ " at step 2-3 and step $3-3$
} 


\subsection{Security}

We first prove the perfect privacy.

Lemma 3. There exists a subset $A \subset\left\{r_{1}, \cdots, r_{t n}\right\}$ such that $|A| \geq \ell$ and the adversary has no information on $A$.

Proof. Consider a non-corrupted channel $i$ such that $i+h n \notin \Lambda_{\mathcal{B}}$. First the sender does not broadcast $r_{i+h n}$ at step 2-1 because $i+h n \notin \Lambda_{\mathcal{B}}$. Next since $f_{i+h n}(i)$ is sent through channel $i$ that the adversary does not corrupt, we have

$$
r_{i+h n}=u_{i+h n, i}=f_{i+h n}(i) .
$$

Further the adversary observes at most $t$ values of $\left(f_{i+h n}(1), \cdots, f_{i+h n}(n)\right)$. Hence the adversary has no information on $r_{i+h n}=f_{i+h n}(i)$ because $\operatorname{deg} f_{i+h n}(x) \leq$ $t$.

Note that the adversary corrupts at most $t$ channels and for each corrupted channel $i$, the adversary gets $r_{i}, r_{i+n}, \ldots, r_{i+(t-1) n}$. Therefore, there exists a subset $A \subset\left\{r_{1}, \cdots, r_{t n}\right\}$ such that

$$
|A| \geq n t-\left|\Lambda_{\mathcal{B}}\right|-t^{2}=n t-k-t^{2}
$$

and the adversary has no information on $A$. Finally

$$
n t-k-t^{2} \geq(2 t+1) t-t-t^{2}=t^{2}=\ell .
$$

Therefore, the adversary has no information on $R(n t+1), \ldots, R(n t+\ell)$ from Sec.4.1. Hence she learns no information on $s_{i}$ for $i=1, \cdots, \ell$.

We next prove the perfect reliability. If $j \notin$ FORGED and $j \notin d_{I}\left(U_{i+h n}, Y_{i+h n}\right)$, then $f_{i+h n}(j)=y_{i+h n, j}=u_{i+h n, j}$ from the definition of $d_{I}\left(U_{i+h n}, Y_{i+h n}\right)$. Therefore,

$$
u_{i+h n}(j)=u_{i+h n, j}
$$

for all $j \in d_{I}\left(U_{i+h n}, Y_{i+h n}\right)$, and for all $j$ such that $j \notin d_{I}\left(U_{i+h n}, Y_{i+h n}\right)$ and $j \notin$ FORGED. This means that $u_{i+h n}(j)=u_{i+h n, j}$ for each $j \in$ (FORGED $\cup$ $\left.d_{I}\left(U_{i+h n}, Y_{i+h n}\right)\right)$, where

$$
\left.\mid \overline{\text { FORGED }} \cup d_{I}\left(U_{i+h n}, Y_{i+h n}\right)\right)|\geq| \overline{\text { FORGED }} \mid \geq n-t=2 t+1-t=t+1 .
$$

Further since $\operatorname{deg} u_{i+h n}(x) \leq t$ and $U_{i+h n} \in \mathcal{C}$, it holds that

$$
\left(u_{i+h n}(1), \cdots, u_{i+h n}(n)\right)=\left(u_{i+h n, 1}, \cdots, u_{i+h n, n}\right) .
$$

In particular, $u_{i+h n}(i)=u_{i+h n, i}$. Therefore from eq.(10), we have that

$$
R(i+h n)=r_{i+h n}=u_{i+h n, i}=u_{i+h n}(i)=R^{\prime}(i+h n)
$$

for each $i+h n$. Hence we obtain that $R^{\prime}(x)=R(x)$ because $\operatorname{deg} R^{\prime}(x) \leq n t-1$ and $\operatorname{deg} R(x) \leq n t-1$. Consequently,

$$
s_{i}^{\prime}=c_{i}-R^{\prime}(n t+i)=c_{i}-R(n t+i)=s_{i} .
$$

Thus the receiver can compute $s_{i}^{\prime}=s_{i}$ correctly for $i=1, \cdots, \ell$. 


\subsection{Efficiency}

Let $\operatorname{COM}(\mathrm{i})$ denote the communication complexity of Step $i$ for $i=1,2$. Note that $\left|d_{u}\left(U_{i+h n}, Y_{i+h n}\right)\right|=\left|d_{I}\left(U_{i+h n}, Y_{i+h n}\right)\right| \leq t$ for each $i+h n$. Then

$$
\begin{aligned}
\operatorname{COM}(1)= & O(t n(n+n)|\mathrm{F}|)=O\left(t n^{2}|\mathrm{~F}|\right), \\
\operatorname{COM}(2)= & O\left(\left(\left|d_{I}\left(U_{i+h n}, Y_{i+h n}\right)\right| \log _{2} n+\left|d_{u}\left(U_{i+h n}, Y_{i+h n}\right)\right||\mathrm{F}|\right) t n \times n\right. \\
& \left.\quad+\left(\log _{2} n+n|\mathcal{B}||\mathrm{F}|+\left|\Lambda_{\mathcal{B}}\right| \log _{2} n\right) n+t^{2}|\mathrm{~F}| n\right) \\
= & O\left(n^{2} t^{2} \log _{2} n+n^{2} t^{2}|\mathrm{~F}|+n \log _{2} n+n^{2} t|\mathrm{~F}|+t n \log _{2} n+t^{2}|\mathrm{~F}| n\right) \\
= & O\left(n^{2} t^{2}|\mathrm{~F}|\right)
\end{aligned}
$$

because $|\mathcal{B}|=\left|\Lambda_{\mathcal{B}}\right| \leq t$. Hence, the total communication complexity is $O\left(n^{2} t^{2}|\mathrm{~F}|\right)$, and the transmission rate is $O\left(n^{2}\right)$ because the sender sends $t^{2}$ secrets.

It is easy to see that the computational costs of the sender and the receiver are both polynomial in $n$.

\section{Final 2-Round PSMT}

The transmission rate is still $O\left(n^{2}\right)$ in the 2-round PSMT shown in Sec.5. In this section, we show how to reduce it to $O(n)$ by using the technique of $[1$, page 406] and [8]. Then we can obtain the first 2-round PSMT for $n=2 t+1$ such that not only the transmission rate is $O(n)$ but also the computational costs of the sender and the receiver are both polynomial in $n$.

\subsection{Generalized Broadcast}

Suppose that the receiver knows the locations of $k(\leq t)$ channels that the adversary forged, and the sender knows the value of $k$. For example, suppose that the receiver knows that channels $1,2, \cdots, k$ are forged. Note that the adversary can corrupt at most $t-k$ channels among the remaining $n-k$ channels $k+$ $1, \cdots, n$.

In this case, it is well known that the sender can send $k+1$ field elements $u_{1}, u_{2}, \ldots, u_{k+1}$ reliably with the communication complexity $O(n|\mathrm{~F}|)$ as follows.

1. The sender finds a polynomial $p(x)$ with $\operatorname{deg} p(x) \leq k$ such that $p(1)=u_{1}$, $p(2)=u_{2}, \ldots, p(k+1)=u_{k+1}$.

2. She sends $p(i)$ through channel $i$ for $i=1, \cdots, n$.

Without loss of generality, suppose that the receiver knows that channels $1, \cdots, k$ are forged by the adversary. Then he consider a shortened code such that a codeword is $(p(k+1), \cdots, p(n))$. The minimum Hamming distance of this code is $(n-k)-k=2 t+1-2 k=2(t-k)+1$. Hence the receiver can correct the remaining $t-k$ errors.

This means that the receiver can decode $(p(k+1), \cdots, p(n))$ correctly. Then he can reconstruct $p(x)$ by using Lagrange formula because

$$
n-k=2 t+1-k \geq 2 k+1-k=k+1 \geq \operatorname{deg} p(x)+1 .
$$

Therefore he can obtain $u_{1}=p(1), \ldots, u_{k+1}=p(k+1)$ correctly. 


\subsection{Matching of Graph}

Let $G=(\mathrm{V}, \mathrm{E})$ be the undirected simple graph with the vertex set $\mathrm{V}$ and the edge set $\mathrm{E}$. A matching of the graph $G$ is an edge set $M \subseteq \mathrm{E}$ such that no two edges in $M$ are connected. A matching $M$ is said to be maximal if there is no matching $M^{\prime} \neq M$ such that $M \subseteq M^{\prime}$.

We can find a maximal matching $M$ of $G$ easily (in polynomial time) by using a greedy algorithm as follows.

1. Let $M=\emptyset$.

2. For each edge $e$ in $\mathrm{E}$, do:

If $e$ is not connected to any edge in $M$, then add $e$ to $M$.

3. Output $M$.

Definition 4. For a vertex $v \in V$, let $\operatorname{deg}_{G}(v)$ denote the number of edges which are connected to $v$. Define

$$
D_{\max }=\max _{v \in V} \operatorname{deg}_{G}(v) .
$$

We then say that $D_{\max }$ be the maximum degree of the graph $G$.

Theorem 3. For a graph $G=(\mathrm{V}, \mathrm{E})$, let $M$ be a maximal matching and $D_{\max }$ be the maximum degree. Then $|\mathrm{E}| \leq 2|M| \cdot D_{\max }$.

Proof. For a maximal matching $M$, define

$$
V(M)=\{v \in V \mid \text { some } e \in M \text { is connected to a vertex } v\} .
$$

Delete all the edges connected to $V(M)$ from $G$. Then from the definition of maximal matching, we have no edges. Further $|V(M)|=2|M|$. Therefore,

$$
|\mathrm{E}| \leq \Sigma_{x \in V(M)} \operatorname{deg}_{G}(x) \leq 2|M| D_{\max } .
$$

In [1, page 406] and [8], a maximum matching was used. Instead we use a maximal matching because it is sufficient for our purpose, and it is easier to find a maximal matching than a maximum matching.

\subsection{How to Improve Step 2-5}

In the 2-round PSMT shown in Sec.5, step 2-5 is the most expensive part, where the sender broadcasts $d_{u}\left(U_{i+h n}, Y_{i+h n}\right)$ and $d_{I}\left(U_{i+h n}, Y_{i+h n}\right)$ for each $i+h n$.

In this subsection, we will show a method which reduces the communication complexity of step 2-5 from $O\left(n^{2} t^{2}|\mathrm{~F}|\right)$ to $O\left(n^{2} t|\mathrm{~F}|\right)$. We modify step $2-5$ as follows.

Step 2. The sender does the following. 
5' For $h=0,1, \cdots, t-1$, do:

(a) Construct an undirected graph $G_{h}=\left(\mathrm{N}, \mathrm{E}_{h}\right)$ such that $(i, j) \in \mathrm{E}_{h}$ if and only if $u_{i+h n, j} \neq y_{i+h n, j}$ or $u_{j+h n, i} \neq y_{j+h n, i} .{ }^{10}$

(b) Find a maximal matching $M_{h}$ of $G_{h}$.

(c) For each edge $e=(i, j) \in M_{h}$,

i. If $u_{i+h n+i, j} \neq y_{i+h n+i, j}$ then broadcast $x_{e}=\left((h, i, j), u_{i+h n, j}, y_{i+h n, j}\right)$.

ii. Else broadcast $x_{e}=\left((h, i, j), u_{j+h n, i}, y_{j+h n, i}\right)$.

(d) Send $\left\{d_{u}\left(U_{i+h n}, Y_{i+h n}\right) \mid i=1, \cdots, n\right\}$ and $\left\{d_{I}\left(U_{i+h n}, Y_{i+h n}\right) \mid i=\right.$ $1, \cdots, n\}$ to the receiver by using the generalized broadcasting as shown below.

If there exists an edge $e=(i, j) \in M_{h}$, then channel- $i$ is forged or channel- $j$ is forged. Therefore,

$$
\left|M_{h}\right| \leq t
$$

from the definition of maximal matching. For each $h$, the communication complexity of step 2-5'(c) is $O(t n|\mathrm{~F}|)$ because $\left|M_{h}\right| \leq t$. For all $h$, the communication complexity is $O\left(n t^{2}|\mathrm{~F}|\right)$

After step 2-5'(c), the receiver can find at least one forged channel from each $x_{e}$, where $e \in M_{h}$. Hence he can find at least $\left|M_{h}\right|$ forged channels from $\left\{x_{e} \mid e \in M_{h}\right\}$ from the definition of maximal matching.

Hence the sender can send $\left|M_{h}\right|+1$ field elements reliably with the communication complexity $O(n|\mathrm{~F}|)$ by using the generalized broadcasting (see Sec.6.1).

Next from Theorem 3, we obtain that

$$
\left|\mathrm{E}_{h}\right| \leq 2\left|M_{h}\right| t
$$

because $\operatorname{deg}_{G_{h}}(i) \leq t$ for all $i$ from step 2-2(a). Further it is easy to see that

$$
\sum_{i=1}^{n}\left|d_{u}\left(U_{i+h n}, Y_{i+h n}\right)\right|=\sum_{i=1}^{n}\left|d_{u}\left(U_{i+h n}, Y_{i+h n}\right)\right| \leq 2\left|\mathrm{E}_{h}\right| \leq 4\left|M_{h}\right| t
$$

Therefore, for each $h$, the sender can send $\left\{d_{u}\left(U_{i+h n}, Y_{i+h n}\right) \mid i=1, \cdots, n\right\}$ and $\left\{d_{I}\left(U_{i+h n}, Y_{i+h n}\right) \mid i=1, \cdots, n\right\}$ to the receiver reliably with the communication complexity $O(n t|\mathbf{F}|)$ by using generalized broadcasting. For all $h$, the communication complexity is $O\left(n t^{2}|\mathrm{~F}|\right)$.

This means that the sender can send all $d_{u}\left(U_{i+h n}, Y_{i+h n}\right)$ and $d_{I}\left(U_{i+h n}, Y_{i+h n}\right)$ reliably with the communication complexity $O\left(n t^{2}|\mathrm{~F}|\right)$.

\subsection{Final Efficiency}

Consequently, we obtain $\operatorname{COM}(2)=O\left(n^{2} t|\mathrm{~F}|\right)$ because the communication complexity of step 2-5' is now reduced to $O\left(n^{2} t|\mathrm{~F}|\right)$. On the other hand, $\operatorname{COM}(1)=$ $O\left(n^{2} t|\mathrm{~F}|\right)$ from Sec.5.3. To summarize,

$$
\operatorname{COM}(1)=O\left(n^{2} t|\mathrm{~F}|\right) \text { and } \operatorname{COM}(2)=O\left(n^{2} t|\mathrm{~F}|\right)
$$

$\overline{10}$ This means that channel- $i$ is forged or channel $-j$ is forged. 
in our final 2-round PSMT. Hence, the total communication complexity is $O\left(n^{3}|\mathrm{~F}|\right)$ because $n=2 t+1$.

Now the transmission rate is $O(n)$ because the sender sends $t^{2}$ secrets which is $O\left(n^{2}|\mathrm{~F}|\right)$. Finally, it is easy to see that the computational costs of the sender and the receiver are both polynomial in $n$.

\section{Acknowledgement}

We would like to thank Jun Furukawa and Adi Shamir for useful discussion.

\section{References}

1. S.Agarwal, R.Cramer and R.de Haan: Asymptotically Optimal Two-Round Perfectly Secure Message Transmission. CRYPTO 2006: pp.394-408 (2006)

2. D.Dolev, C.Dwork, O.Waarts, M.Yung: Perfectly Secure Message Transmission. J. ACM 40(1): pp.17-47 (1993)

3. Y.Desmedt, Y.Wang and M.Burmester: A Complete Characterization of Tolerable Adversary Structures for Secure Point-to-Point Transmissions Without Feedback. ISAAC 2005: pp.277-287 (2005)

4. M.Fitzi, M.Franklin, J.Garay, S.Vardhan: Towards Optimal and Efficient Perfectly Secure Message Transmission. TCC 2007: pp.311-322 (2007)

5. M.Hirt, U.Maurer: Player Simulation and General Adversary Structures in Perfect Multiparty Computation. J. Cryptology 13(1): pp.31-60 (2000)

6. M.V.N.A.Kumar, P.R.Goundan, K.Srinathan, C.P.Rangan: On perfectly secure communication over arbitrary networks. PODC 2002: pp.193-202 (2002)

7. H.Md.Sayeed and H.Abu-Amara: Efficient Perfectly Secure Message Transmission in Synchronous Networks. Inf. Comput. 126(1): pp.53-61 (1996)

8. K. Srinathan, Arvind Narayanan, C. Pandu Rangan: Optimal Perfectly Secure Message Transmission. CRYPTO 2004: pp.545-561 (2004) 